\title{
Téoros
}

Revue de recherche en tourisme

\section{Une fête ou un événement réussi, ça se prépare!}

\section{Françoise Labarre et Jacques Renaud}

Volume 5, numéro 2, juillet 1986

Tourisme, fêtes et événements

URI : https://id.erudit.org/iderudit/1080576ar

DOI : https://doi.org/10.7202/1080576ar

Aller au sommaire du numéro

Éditeur(s)

Université du Québec à Montréal

ISSN

0712-8657 (imprimé)

1923-2705 (numérique)

Découvrir la revue

Citer cet article

Labarre, F. \& Renaud, J. (1986). Une fête ou un événement réussi, ça se prépare! Téoros, 5(2), 26-28. https://doi.org/10.7202/1080576ar d'utilisation que vous pouvez consulter en ligne.

https://apropos.erudit.org/fr/usagers/politique-dutilisation/ 


\title{
Une fête ou un événement réussi, ça se prépare!
}

\author{
par Françoise Labarre et Jacques Renaud*
}

Une féte populaire ou un événement c'est, à la base, une personne, ou une association qui a une idée et qui veut la réaliser dans un temps limité. Cette personne, ou association recherchera des alliés avec lesquels elle donnera une forme à l'idée, puis tentera de l'inscrire dans un contexte social afin de la faire devenir réalité. C'est ce qu'on appelle avoir un projet.

Vue sous cet anglè, toute fête (ou événement) est un projet. Et une de ses principales particularités, c'est que dès sa naissance, on saura à quel moment il s'achèvera. Décider de son début, c'est aussi décider de sa fin. C'est lả que réside le défi.

Pour les promoteurs et les réalisateurs de projets, le plaisir de travailler dans un tel contexté résidera dans la proximité du but a atteindre; en effet. la force de travail investie trouvera à brève échéance sa consécration dans le produit réalisé (la fête, l'évênement) et dans ses résultats évidents (l'appréciation du public). Le produit cherchera habituellement à être original, nouveau, inusité et fera donc appel à la création et à l'innovation.

Parce qu'il s'agit d'innover, voire de créer, on croit souvent qu'il n'y a pas de méthode, qu'il $n^{4} y$ a pas de savoir-faire existant et qu'il faut à chaque fois reinventer la roue.

Cependant, l'expérience a permis de constater que les promoteurs, les décideurs, les coordonnateurs et les réalisateurs de projet se butent souvent aux mêmes difficultés, originant la plupart du temps d'une vue d'ensemble incomplète des dimensions que comporte l'organisation d'un projet.

Pour agir efficacement dans un projet, il faut en saisir les trois (3) dimensions complémentaires: la mécanique, c'est-à-dire les étapes chronologiques nécessaires à sa réalisation, la dynamique, c'est-ầ-dife les

\footnotetext{
"Françoise Labarre et Jacques Renaud sont deux praticiens de lorganisation qui ont oeuved au sein de nombreux comités organisateurs d'evenements majeurs au Quebec. Its offrent un programme de formation en ges. tion de projet intitule "Comment reussir un projet e! devenif mattre de son organisation"
}

rapports d'équilibre et de pouvoir évoluant dans des structures hiérarchisées et enfin le facteur humain, c'est-à-dire les facteurs de motivation, de productivité et de ralliement. C'est la maitrise de ces trois (3) dimensions qui garantit le succès d'un projet.

Cet article présente un guide pratique permettant de franchir les étapes nécessaires à la réalisation d'un projet:

- l'étape indispensable: définir le sens du projet;

- l'étape déterminante: formuler le concept du projet;

- l'étape décisive: élaborer le plan directeur:

- l'étape de croissance: bătir une organisation;

- l'étape vitale: créer une dynamique;

- l'étape préventive: évaluer l'avancement du projet;

- l'étape finale: réussir.

\section{L'étape indispensable: définir le sens du projet}

Définir le sens du projet, c'est ni plus ni moins que la mise en commun des aspirations, des motivations et des intérêts des promoteurs. Ce travail d'équipe a pour but de ramasser dans un document interne les éléments qui serviront à la formulation du projet. Ce document détermine l'espace de réalisation du projet.

C'est une étape indispensable pour partir le projet sur des bases solides et communes, même si parfois devant l'urgence d'agir ou la non-complicité des promoteurs, on peut vouloir passer outre cette première étape.

La réflexion et la discussion pour définir le sens du projet se fera à partir de la démarche suivante:

- Visualiser la finalité du projet, c'est-àdire imaginer le projet à réaliser, identifier les résultats, les retombées souhaitées et l'impact social, déterminer l'essentiel du projet en termes de qualité, de rentabilité et de fonctionnement de l'organisation.
- Cerner les motivations à l'investissement, c'est-à-dire identifier les motivations personnelles et professionnelles à court, moyen et long termes des promoteurs et percevoir leurs complémentarités.

- Identifier les réalités hors-projet, c'està-dire identifier les conditions de réalisation fixées par d'autres qui peuvent avoir des effets facilitants ou empéchants.

\section{L'étape déterminante: \\ formuler le concept du projet} Formuler le concept du projet, c'est en quelque sorte en déterminer la personna. lité. Cette étape est déterminante, donnant lieu à un document d'orientation qui sera présenté aux instances décisionnelles (le conseil d'administration, le comité exécutif, etc.). L'acceptation du concept permettra d'obtenir, s'il y a lieu, les budgets de démartage nécessaires à la mise en route du projet.

Le concept est donc un document d'orientation qui doit contenir l'essentiel du projet à réaliser et des moyens requis pour le faire. On y retrouvera des éléments déjà définis lors de la première étape et on y ajoutera les éléments de contenu et d'organisation. Cela suppose que les promoteurs ont déjà de bonnes raisons d'étre assurés que le projet pourrait avoir lieu et qu'il serait bien reçu par la clientèle visée.

Dans le concept, on retrouvera:

- une analyse du contexte précisant le besoin auquel le projet veut répondre;

- le but et les objectifs du projet.

- le cadre de réalisation: les dates, les lieux de réalisation, les publics visés, les conditions de réalisation, les facteurs infligeants:

- la description des activités du projet: les caractéristiques recherchées et la programmation proposée;

- la structure d'organisation: les táches à accomplir, l'organigramme, les ressources matérielles nécessaires:

- l'échéancier: les dates charnières:

- le budget préliminaire: les revenus et les grandes catégories de dépenses prévisibles; 


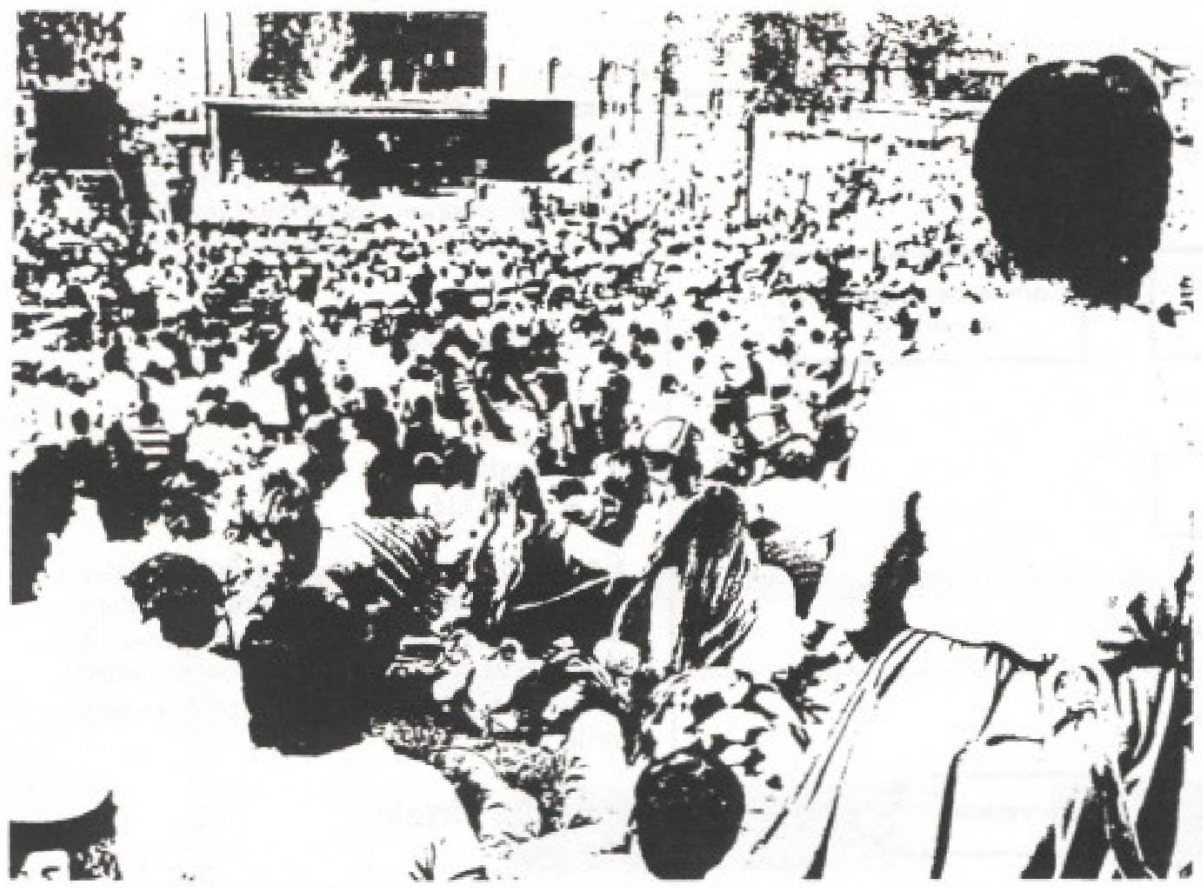

Une fêto, un ávénement, c'est d'abord un projet qui comme tout autre doit être bien préparé si on veut qu'il soit réussi.

- les retombées économiques et sociales escomptées, les contraintes et les mises en garde s'il y a lieu.

On devrait s'en tenir à un document cour et explicite ne dépassant pas dix (10) pages qui, une fois adopté, deviendra l'outil de référence durant toute la durée du projet pour tous les collaborateurs-clés.

\section{L'étape décisive: \\ élaborer le plan directeur}

Après l'étape de la formulation du concept et son adoption, vient l'étape de la mise en forme et de la réalisation du projet. Cette étape comprend cinq (5) phases qui se dérouleront consécutivement: l'exploration, la planification, la préparation, l'exécution et l'évaluation du projet.

\section{Lexploration}

L'exploration permet de vérifier et de mesurer la faisabilité technique, financière et sociale du projet. C'est une phase de recherche et d'études de faisabilité. Ces études préciseront les paramètres internes du projet en termes de qualité, de coûts, de ressources humaines et matérielles et d'échéancier. Elles préciseront aussi les interrelations à établir avec les intervenants externes concernés par le projet (services de sécurité, services municipaux, souscontractants, producteurs délégués, etc.) Cette phase orientera le plan directeur.

Les dossiers qui feront l'objet d'études de faisabilité sont ceux qui ont une implication technique et financière importante en fonction du but et des objectifs du projet.

\section{La planification}

Planifier, c'est prévoir! C'est aussi rechercher, choisir et préparer ce que l'on veut faire ou réaliser. C'est durant cette phase que naïtra le plan directeur des opérations du projet.

Le plan directeur est un document généralement assez volumineux qui doit être révisé quand les conditions connues et existantes lors de son élaboration sont modifiées. Ce document sera utile aux gestionnaires et aux responsables opérationnels durant toute la durée du projet.

On y retrouvera:

- un résumé du concept;

- le programme d'activités;

- la structure d'organisation;

- le budget detaillé:

- l'échéancier détaillé.

Ce document sera sous la responsabilité du directeur général du projet et présenté aux instances décisionnelles. Il aura fait l'objet de consultations auprès de tous les interd'être associés à l'exécution du projet.

\section{La préparation}

S'appuyant sur le plan directeur, cette phase sera celle de la mise en place des ressources humaines et matérielles nécessaires, ainsi que des plans spécifiques d'opérations et des outils de gestion pertinents. Cette phase sera donc consacrée à:

- l'organisation de l'espace de travail:

- la formulation des politiques administratives:

- la mise en place des équipes de travail:

- la détermination des objectifs et activités spécifiques:

- l'établissement des devis techniques:

- l'élaboration d'un cahier opérationnel.

\section{L'exécurion}

C'est la phase de la réalisation concrète du but du projet. Durant cette phase, des rapports d'étape devront ếtre présentés aux venants internes et externes susceptibles instances décisionnelles, dans un but préventif plutôt que curatif. On concentrera l'énergie des équipes de travail:

- à la coordination du déroulement par un suivi du cahier opérationnel en fonction de priorités d'action:

- au contrôle budgétaire des dépenses en fonction des revenus:

- au règlement rapide des situations d'urgence grăce à des mécanismes de décision légers et efficaces.

L'évaluation

L'evaluation est la mesure des résultats du projet. Elle contribue à l'avancement du savoir et au développement de l'organisme promoteur et des membres du comité orga" nisateur.

Dans la phase d'évaluation, on retrouvera:

- le rapport des activités réalisées mesurées qualitativement et quantitativement en regard du plan directeur;

- le bilan financier présentant l'état final des revenus et des dépenses du projet:

- l'étude des impacts ou retombées économiques et sociales sur la collectivité bénéficiaire du projet et sur l'organisme promoteur.

\section{L'étape de croissance: bâtir une organisation}

Bâtir l'organisation d'un projet, c'est bâtir l'organigramme du projet et en déterminer le mode de gestion.

Un organigramme ou un mode de gestion déficients entrainent inévitablement des difficultés de parcours parfois dramatiques. pour la survie du projet. L'organigramme se résume habituellement à quatre (4) ou cinq (5) grands secteurs d'opérations (voir page suivante). L'organigramme vient en quelque sorte déterminer le rôle des joueurs et le mode de gestion, et en fixer les règles du jeu.

Bâtir un organigramme c'est:

- choisir la forme juridique de l'entreprise et la structure d'organisation;

- identifier les secteurs d'activités (regroupement logique des responsabilités de dossiers de même nature) et les responsabilités de dossiers (ensemble des éléments constituant une tấche distincte);

- affecter les niveaux d'autorité c'est-àdire déterminer les niveaux de décision (décideurs), de coordination (gestionnaires) et d'exécution (réalisateurs)

Déterminer le mode de gestion, c"est:

- définir les méthodes de travail:

- définir les moyens pour assurer la circulation de l'information à l'intérieur de l'organisation. 


\section{Organigramme-type d'un projet}

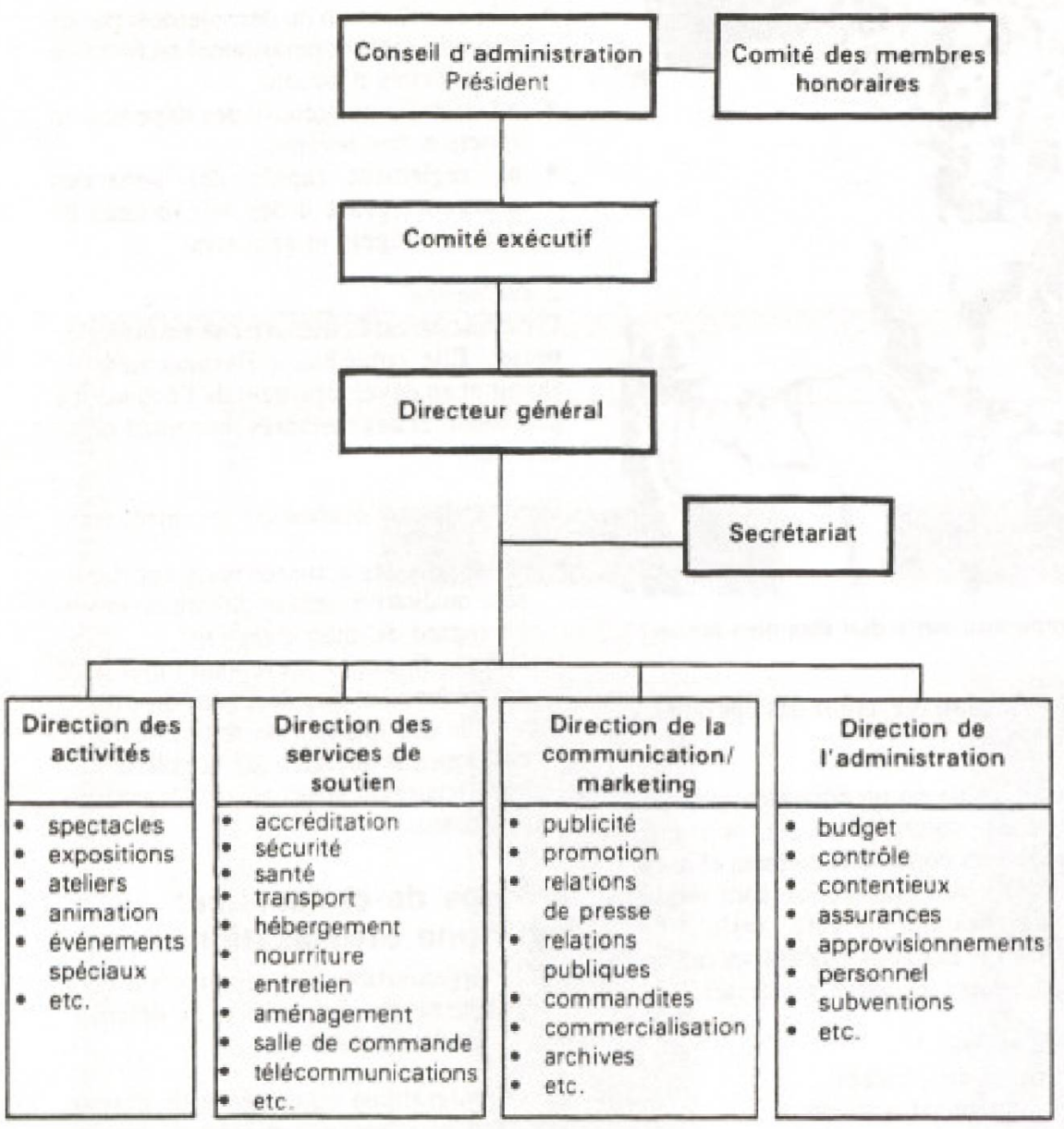

L'étape vitale:

\section{créer une dynamique}

Créer une dynamique, c'est d'abord se préoccuper de la motivation du personnel de l'organisation, et permettre l'expression de ses valeurs dominantes: le goût du travail bien fait, du défi, du dépassement, le goût de participer, de se développer personnellement, d'étre reconnu et I'horreur d'être manipulé. Une organisation motivatrice visera donc à fournir à chaque individu toute l'information nécessaire à son travail, à maintenir un état d'esprit enthousiaste envers le rendement à fournir, et à accorder la considération et les récompenses appropriées aux réalisations individuelles. Elle visera aussi à encourager la fixation individuelle d'objectifs favorisant la création et l'innovation. Ceci implique un leadership qui sait rallier les équipes du projet autour de son sens premier, qui encourage plutôt qu'il ne punit, qui fait appel à la conjugaison des idées et des influences de chacun (décentralisation), et qui favorise les relations entre la tềte et la base de l'organisation.

Creerer une dynamique, c'est aussi avoir une organisation ouverte en impliquant des intervenants du milieu immédiat du projet: par l'information, l'implication concrète dans certains dossiers, la participation à des comités spécifiques. Ainsi impliqués, les intervenants du milieu coopéreront d'autant mieux lors de l'exécution du projet qu'ils considéreront que le projet leur appartient un peu.

Enfin, créer une dynamique, c'est aussi avoir la préoccupation des "clients", ceux qui en bénéficieront. Associer le public à l'élaboration de certaines activités, susciter leur participation et tester certaines activités auprès d'eux est une garantie d'appropriation du projet lors de sa réalisation. Ainsi, le projet offrira des activitês adaptées aux publics visés et ces derniers s'y sentiront associés.

\section{L'étape préventive:}

\section{évaluer I'avancement du projet}

Tout projet évolue rapidement et peut parfois, malgré une bonne planification, prendre des directions nouvelles ou inusitées. $C^{\prime}$ est pourquoi il est essentiel de prévoir des temps d'arrét en cours de route.
Ces temps d'arrêt sont pour les instances décisionnelles des temps de synthèse et de décision durant lesquels le projet est réévalué en fonction des facteurs qui déterminent habituellement l'avancement ou non d'un projet.

Ces facteurs-clés sont:

- le temps;

- l'argent;

- la qualité;

- la visibilité.

Ces temps d'arrét doivent se faire régulièrement à la fin des étapes importantes (dates charnières) ou selon les besoins (situations d'urgence) et permettent la réorganisation du projet s'il y a lieu en fonction de sa nouvelle réalité.

\section{L'étape finale: réussir}

La compréhension et l'appropriation des six étapes sommairement décrites entrainent en principe la réussite d'un projet. Mais audelà des méthodes et techniques, la réussite d'une fête ou d'un événement repose aussi sur la qualité des organisateurs et sur leurs motivations profondes à accomplir le projet.

L'organisation d'une fête ou d'un événement est un champ professionnel qui s"est beaucoup développé au cours des vingt dernières années au Québec. Il regroupe des tas d'individus un peu marginaux, en mal de réalisation, remplis d'idéaux sociaux et personnels, qui font preuve d'un dynamisme remarquable au travail. Peu d'experts dans le domaine, mais beaucoup d'organisateurs et d'organisatrices de bonne volonté qui par leur courage et leur détermination réussiront souvent de véritables prouesses conceptuelles, organisationnelles et administratives.

\section{Réussir un projet, c'est d'abord y croire!}

En plus d'y croire, il faut prendre conscience qu'une fête ou un évênement est un tout qui comporte une dynamique qui lui est propre. Un projet vivant évolue constamment, parfois dans des chemins que la logique est inapte à prévoir. C'est là qu'intervient la qualité essentielle de l'organisateur-trice qui constitue à maintenir le cap sur le but initial du projet utilisant, pour ce faire, son jugement, son instinct et son inspiration.

Cette qualité 5 acquiert en intégrant de façon personnelle la logique de l'organisation de manière à utiliser les trois (3) dimensions complémentaires selon l'évolution et le contexte de réalisation de sa fête ou de son événement.

Réussir un projet c'est aussi être maître de son organisation! 